\title{
DOSSIER
}

\section{FLORENT PESTRE}

Université Paris-Sud, Laboratoire PESOR
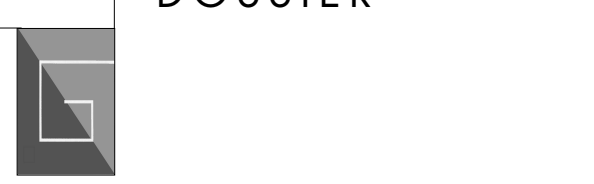

\section{Construire une stratégie de responsabilité sociale de la firme multinationale}

\section{Le cas du groupe Lafarge}

L'objectif de cet article est de proposer un modèle de construction de stratégie de responsabilité sociale de la firme multinationale. La complexité du fonctionnement interne de ce type d'entreprise est appréhendée par une analyse des relations siège-filiales. En effet, cette recherche rapproche deux champs distincts : la littérature portant sur les processus stratégiques d'une part, et celle liée aux stratégies internationales d'autre part. Une étude de cas retrace les différentes phases du processus de formulation et de mise en œuvre de la stratégie de lutte contre le sida du groupe Lafarge en Afrique. La conceptualisation de ce phénomène aboutit à la discussion d'un processus transnational de co-construction de la stratégie de RSE par le siège et les filiales. 
I est important de s'intéresser à la question de la mise en œuvre des stratégies de responsabilité sociale de l'entreprise (RSE) car des enjeux humains sont rattachés à son exécution. En effet, dans un contexte international, la firme doit justifier le rôle positif qu'elle joue face aux problèmes de violations des droits de l'homme, de pauvreté, de malnutrition ou de maladies. En l'absence de normes mondiales, il est toutefois difficile de savoir quelles stratégies concevoir et quels outils utiliser pour les mettre en œuvre (Gordon, 2001). L'objectif de cet article est de contribuer à une meilleure compréhension du phénomène de construction de ces stratégies par les firmes multinationales. Cette problématique rejoint deux thématiques centrales et majeures en management international, les stratégies internationales et les relations siège-filiales, qui sont utilisées comme angle d'attaque théorique dans cette recherche.

La première partie de ce papier souligne l'intérêt de s'intéresser à la construction de stratégies dans la firme multinationale selon une perspective processuelle. En effet, la mobilisation des travaux portant sur les processus stratégiques permet de reconsidérer la complexité des firmes multinationales et de sortir le champ de recherche relatif aux relations siège-filiales de l'impasse dans laquelle il se situe depuis une vingtaine d'années. La deuxième partie de l'article dévoile la logique de construction de la stratégie de lutte contre le sida de l'entreprise Lafarge en Afrique du Sud. Une narration décrit le phénomène observé grâce aux données recueillies auprès de la direction générale du groupe et auprès d'acteurs locaux dans la filiale sud-africaine. Enfin, la troisième partie s'appuie sur ce matériau empi- rique et esquisse un modèle transnational de construction de stratégies de RSE. Celui-ci se distingue d'un processus strictement ascendant, initié par la filiale et d'un processus exclusivement descendant, impulsé par le siège. Sont enfin discutées les relations siège-filiales entretenues tout au long de chacune des phases de ce processus.

\section{I - STRATÉGIES INTERNATIONALES, RELATIONS SIÈGE-FILIALES ET RSE}

Les firmes multinationales sont des organisations particulièrement complexes car elles sont confrontées à des situations et à des problèmes bien plus variés que ceux que les plus grandes firmes domestiques peuvent rencontrer (Egelhoff, 1991). Pour comprendre comment se développent leurs stratégies, il est nécessaire d'examiner leur fonctionnement interne. Or le principal canal qui permet à l'entreprise de gérer et de contrôler ses stratégies et ses activités est, comme le rappelle Otterbeck (1981), les relations siège-filiales. Cette première partie justifie l'intérêt d'adopter une perspective processuelle des relations siègefiliales pour mieux comprendre la formation et le déploiement des stratégies de RSE.

\section{1. Évolution des firmes multinationales : vers une approche processuelle des relations siège-filiales}

Dans un article paru en 2008, Ghemawat alerte la communauté scientifique sur le peu de travaux consacrés aux stratégies internationales. Un an plus tard, Pisani (2009) montre que seulement cinq pour cent des articles publiés sur la période de 2002 à 2006 dans les vingt meilleures revues scientifiques traitent d'un sujet de management 
international. Parmi ces articles, seulement douze pour cent étudient les politiques et stratégies des firmes multinationales. Pour tenter de comprendre les raisons de cette relative impasse dans lequel se situe ce champ de recherche, il est nécessaire d'examiner et de prendre en compte les évolutions récentes des formes d'organisations multinationales.

La question centrale sous-jacente à la construction de stratégies internationales est celle qui concerne l'équilibre entre le besoin d'intégration globale et de standardisation d'une part, et le besoin de différenciation et de réactivité aux environnements locaux, d'autre part (Prahalad et Doz, 1987). Les tensions qui s'exercent sur la firme multinationale influencent sa structure organisationnelle et le choix de ses stratégies, qu'elles soient locales, globales ou transnationales. La littérature portant sur les relations siègefiliales se focalise précisément sur les rôles respectifs du siège et des filiales dans la formulation et la mise en œuvre de ces stratégies. Cependant, le paradigme dominant ces recherches est principalement issu d'une conception dyadique et hiérarchique selon laquelle la filiale est une entité instrumentale agissant sur les instructions du siège. Celui-ci pilote, coordonne et contrôle les activités de la filiale par une série de mécanismes principalement formels (Martinez et Jarillo, 1989). La plupart des travaux mobilise en effet la théorie de l'agence et de la contingence et inscrit les relations siègefiliales dans un cadre centre/périphérie (Goold et Campbell, 1987). Or, ce qui caractérise l'évolution des travaux de recherche sur la firme multinationale des vingt dernières années, c'est certainement l'intérêt croissant porté à l'étude des filiales. Ainsi, se sont développés des travaux centrés sur les rôles et les stratégies des filiales, des réseaux constitués par les filiales, ainsi que sur les ressources et les capacités développées par ces dernières (Birkinshaw et Hood, 1998). La filiale étant considérée comme faisant partie d'une série d'unités organisationnelles interdépendantes, elle ne peut plus être perçue comme un simple agent du siège (O’Donnell, 2000).

L'évolution des formes organisationnelles des firmes multinationales (« hétérarchies », « transnationales », " entreprises en réseau », " méta-nationales », etc.) rend caduque l'approche hiérarchique et statique des relations siège-filiales. Quant aux travaux centrés sur la filiale, ils négligent le rôle du siège (Beddi, 2008). Une perspective dynamique s'avère donc intéressante pour ouvrir la boîte noire que constituent les relations multicentres des firmes multinationales. Pour saisir la dynamique des interactions entre les initiatives émergentes issues des capacités de la filiale et la valeur ajoutée du siège, il semble opportun d'utiliser une approche processuelle des engagements stratégiques. Celle-ci trouve ses origines dans les travaux de Simon, March et Cyert de l'école de Carnegie Mellon. Elle a été développée par Joseph Bower à la Harvard Business School, ainsi que par Ackerman. Ces travaux se sont fondés sur les firmes multi-divisionnelles comme champs d'étude, y compris pour les travaux plus récents de Burgelman portant sur les stratégies d'innovation. De manière très surprenante, ce domaine de recherche n'a pas été utilisé dans le domaine du management international. Certes, une école processuelle des relations siège-filiales des firmes multinationales s'est développée dans le milieu des années 1980 mais elle s'est construite sur une base conceptuelle 
différente. Il s'agit ainsi des travaux de Prahalad, Doz, Ghoshal, Bartlett, Hedlund et Rolander, centrés autour des tensions entre réactivité locale et efficacité intégrée. L'ambition de cette recherche est de développer les premières briques d'une conceptualisation des processus stratégiques à l'œuvre au sein de la firme multinationale. Le choix s'est porté sur l'étude des stratégies de RSE dans la mesure où le besoin d'une cohérence globale et celui d'une adaptation locale se font tous les deux fortement sentir. Il ne s'agit pas, en effet, d'une activité strictement nécessaire, au sens opérationnel du terme, ce qui permet un jeu plus riche entre siège et filiales, autour de sa définition et de son adoption.

\section{Stratégies de RSE locale, globale et transnationale}

La grande majorité des travaux considère la responsabilité sociale de l'entreprise dans un cadre national bien défini et élude ainsi la question de la responsabilité de la firme multinationale dans les multiples contextes dans lesquels elle opère: les pressions des parties prenantes sont plus nombreuses et leurs attentes plus variées que dans un contexte strictement domestique. Se pose alors la question de savoir quel type de stratégie développer: une stratégie globale, standardisée et uniforme au sein de la firme, ou une stratégie locale et adaptée aux circonstances et aux besoins de chaque communauté dans laquelle s'inscrivent les filiales. Husted et Allen (2006) distinguent ainsi les problématiques de RSE globales qui se définissent relativement à des valeurs et à des règles sociales universelles, des problématiques de RSE locales qui répondent aux spécificités des parties prenantes de chaque pays. Logsdon et Wood (2002) et Arthaud-Day (2005) défendent l'idée d'une stratégie intermédiaire, hybride ou transnationale, qui permet de reconnaître l'existence de principes fondamentaux de RSE sans exclure l'adaptation, lorsque celle-ci est nécessaire. En suivant une logique de RSE locale, la firme prend en effet le risque de gérer des stratégies multiples, dispersées et fragmentées (Meyer, 2004). À l'inverse, une stratégie de RSE globalement intégrée ne laisse aucune marge de manœuvre puisque c'est l'harmonisation de la mise en œuvre de la stratégie qui est recherchée au détriment de son interprétation et de son adaptation. Les conséquences humaines de ce type de choix sont particulièrement fortes: les répercussions se mesurent en termes de vie ou de mort lorsqu'il s'agit par exemple de la santé des salariés, des conditions de travail des sous-traitants, d'accès à l'eau potable ou à l'alimentation de populations locales particulièrement sensibles aux actions de l'entreprise dans les pays pauvres. Ces questions forment de véritables dilemmes pour les dirigeants internationaux qui sont confrontés aux critiques de relativisme éthique et à celles d'impérialisme éthique (Donaldson, 1996).

Si cette phase de réflexion, qui porte sur le contenu de la stratégie de RSE, représente un enjeu de taille pour la firme multinationale - les intérêts de la filiale et ceux du groupe peuvent diverger - la mise en œuvre effective de la stratégie définie soulève des questions auxquelles la littérature existante ne fournit pas de réponses. Plusieurs auteurs, tant en RSE (Capron et Quairel, 2007) qu'en stratégie (Martinet et Reynaud, 2004; Martinet et Payaud, 2008), font état du manque patent de recherches portant sur l'enjeu managérial et organisationnel de 
construction de stratégies de RSE. À l'exception du récent travail de Persais (2010) portant sur la « glocalisation » des pratiques de RSE, il n'existe aucune démarche empirique illustrant le développement des trois types de stratégies rencontrées à l'international (locale, globale et transnationale). L'objectif de cet article est de contribuer à cette question opérationnelle en s'appuyant sur l'examen approfondi d'un cas réel. La deuxième partie de cette recherche livre une description de la démarche de lutte contre le sida développée par Lafarge en Afrique. La monographie s'attache à reconstituer et à retracer, de manière chronologique, les différentes phases de construction de la stratégie, de sa naissance à sa généralisation; elle met systématiquement en parallèle le rôle du siège avec celui de la filiale sud-africaine à chacune des étapes du processus observé.

\section{MÉTHODOLOGIE}

L'objectif de cette recherche est de reconstituer, par le biais d'une étude de cas (Yin, 2003), les différentes phases de construction d'une stratégie de responsabilité sociale du leader français des matériaux de construction, Lafarge. Le groupe fait partie des firmes françaises considérées comme pionnières en matière de développement durable et de RSE (Aggeri et al., 2005). L'étude concerne le projet de lutte contre le sida mis en œuvre dans les filiales africaines au début des années 2000 et s'appuie sur une trentaine d'entretiens menés en français auprès de dirigeants de Lafarge à Paris et en anglais auprès d'acteurs locaux en Afrique du Sud, à Johannesbourg, à Lichtenburg ainsi qu'à Durban. Les entretiens, d'une durée moyenne d'une heure trente, enregistrés et intégralement retranscrits, ont été complétés par des documents secondaires internes (accès à l'Intranet, aux comptes rendus de réunions, etc.) et externes à l'entreprise à des fins de triangulation. Des entretiens avec des acteurs indépendants à l'entreprise (ONG Care, experts de la lutte contre le sida en Afrique du Sud) complètent le dispositif.

\section{Précautions d'interprétation}

La narration proposée dans la partie suivante offre un récit utile à la reconstitution du phénomène observé. Toutefois, son ambition n'est pas de livrer un regard critique sur le comportement de l'entreprise en matière de responsabilité sociale, ni d'évaluer ses choix. En second lieu, bien qu'un seul cas ait des limites certaines, le but de l'étude n'est pas de confirmer ou de tester des modèles théoriques existants mais de mieux comprendre un phénomène particulier et de dégager, à partir d'observations empiriques, une esquisse de modèle de construction de stratégies de RSE. Deux pistes de recherche s'avèrent nécessaires et prometteuses pour mettre en relief les critères de réussite du processus: l'étude de cas d'échec permettrait de mieux faire apparaitre les principaux points d'achoppement lors des quatre phases du processus proposé; l'étude des différents jeux de pouvoir ainsi que de la potentielle instrumentalisation de la RSE par les acteurs rendrait compte de la complexité d'un processus qui est, dans cette recherche, volontairement simplifiée pour répondre à l'objectif de construction théorique de l'article. 


\section{II - LA CONSTRUCTION D'UNE STRATÉGIE DE LUTTE CONTRE LE SIDA}

En 2001, Bertrand Collomb, le président du groupe Lafarge, célèbre l'acquisition de son concurrent anglais, Blue Circle. Cette prise de contrôle scelle l'entrée de l'entreprise dans sept pays de l'ouest africain et renforce sa présence en Afrique du Sud et au Cameroun. Lors de son premier déplacement en Afrique, quelques semaines après l'acquisition, le président s'interroge sur la façon d'intégrer ces nouvelles unités et se rend alors compte de l'enjeu humain et social que représente le VIH/sida. Il prend conscience de l'existence d'un véritable dilemme éthique auquel son entreprise est confrontée car les prix des médicaments anti-rétroviraux, la seule solution pour éviter la mort aux employés malades, sont excessivement élevés. On ne sait pas à quel point l'entreprise est touchée et il n'existe pas de solutions évidentes à proposer. Bertrand Collomb énonce alors deux principes de base à respecter dans l'approche du problème du sida: la confidentialité et la nondiscrimination envers les employés. C'est à cette époque, en 2001, que Frédéric de Rougemont est nommé directeur général de Lafarge en Afrique du Sud. Confronté à la pandémie du sida sur le lieu de travail, il se sent un peu désarmé et sans ressources face à l'étendue du fléau. Il décide de faire du problème du sida une priorité stratégique pour la filiale et il impulse une approche structurée pour faire face à l'épidémie. Il dispose alors de très peu d'informations sur la situation réelle et il se retrouve dans une situation d'incertitude très forte. Il demande à son équipe de direction d'élaborer un programme de lutte contre le sida orienté vers la performance.
L'année suivante marque une étape supplémentaire pour le groupe Lafarge dans son approche de la lutte contre la maladie. En 2001-2002, il s'engage progressivement dans une réflexion pour déterminer le cadre dans lequel développer des solutions; il cherche de l'aide et des exemples dans son environnement externe. En 2002, un partenariat de long terme est créé entre Lafarge et l'ONG internationale Care. Ce partenariat, basé sur l'apprentissage réciproque, aide le groupe à mettre en forme ses actions contre le sida et à formaliser son approche. Lafarge s'engage dans des réseaux professionnels et devient un membre actif de la Coalition mondiale des entreprises (CME) contre le sida et du PIA (Private Investors for Africa). Sur le terrain, la première action menée par la filiale sud-africaine est l'organisation de réunions dans tout le pays, dans chaque bureau, cimenterie ou carrière de l'entreprise. Un consultant externe, expert en matière de gestion du sida sur le lieu de travail, implique les employés ainsi que le principal organisme syndical sudafricain, le COSATU (Congress of South African Trade Unions), dans l'élaboration d'un programme pertinent pour Lafarge en Afrique du Sud. La communication, la consultation et l'implication des employés ne sont toutefois pas choses facile et de nombreux obstacles surgissent: $50 \%$ des travailleurs sont illettrés; 11 langues officielles cohabitent et un climat de suspicion plane de la part de la population noire visà-vis des motivations de la direction de l'entreprise, en raison des souvenirs de la période d'apartheid.

En 2002, la direction générale de Lafarge décide de lancer un projet pilote en Afrique avec l'aide et l'implication de la direction de la zone. La première étape consiste à ras- 
sembler et à collecter les initiatives et les actions existantes grâce à des questionnaires envoyés aux filiales africaines. Ensuite, le groupe décide d'évaluer les coûts engendrés par la maladie dans ses filiales africaines comme l'indique le communiqué suivant: " L'École de santé publique de l'université de Boston a rétrospectivement analysé la répartition des coûts engendrés par un collaborateur atteint du $\mathrm{VIH} /$ sida dans la filiale de Lafarge en Ouganda, avant le lancement du plan d'action intégrant l'accès aux tri-thérapies. L'estimation du coût annuel engendré par une personne vivant avec le VIH/sida, non soignée, est de 4600 euros environ. Outre les coûts visibles que sont l'absentéisme pour $7 \%$, les dépenses de santé pour $3 \%$ et les frais liés aux funérailles pour $5 \%$, l'étude a mis en lumière les coûts cachés: la baisse de la productivité pour $29 \%$, la formation et le recrutement pour $29 \%$, et le temps mobilisé pour s'occuper du sujet pour $27 \%$ \% (communiqué de presse de Lafarge du 11 mai 2005). À partir de ce référentiel, la direction générale de Lafarge demande à chacune de ses filiales d'évaluer et de mesurer précisément les coûts induits du sida sur leurs activités. De son côté, la filiale sud-africaine met en place un comité chargé des problèmes de VIH/sida placé sous l'autorité du directeur des ressources humaines. Ce comité regroupe le directeur de la communication ainsi que des directeurs opérationnels. Le prérequis est que chaque directeur, qui peut être confronté au problème du sida, doit être préparé à gérer ses employés directement touchés par la maladie et à intégrer ces préoccupations dans le fonctionnement quotidien de ses opérations. Le comité définit la politique, planifie les actions et rend des comptes au directeur général. Le directeur des ressources humaines fait aussi partie du comité santé Afrique; à l'échelle de la région, il est ainsi en contact avec les autres filiales africaines du groupe.

La période 2002-2003 représente pour le groupe Lafarge la transformation de l'enjeu éthique que représente le $\mathrm{VIH} /$ sida en enjeu économique. Gérer le VIH/sida devient un facteur clé de succès dans l'activité. La direction de la région Afrique joue un rôle prépondérant lorsqu'elle s'approprie véritablement le problème et s'aperçoit que le sida devient un enjeu économique. La direction générale de Lafarge décide de renforcer et d'étoffer ses ressources consacrées à la lutte contre le sida en embauchant à temps complet un chargé de mission VIH/sida. La nomination de ce « champion interne » se fait en juin 2002 au sein du département des politiques sociales du groupe. Un comité santé Afrique est créé à la même époque et constitue le fer de lance du programme de lutte contre le sida. Avec à sa tête le président de la région Afrique, le comité regroupe un représentant du département des politiques sociales, les dirigeants des filiales africaines, un médecin et un représentant de l'ONG Care. Les initiatives de la filiale sud-africaine se poursuivent avec le développement de campagnes de dépistage. En effet, pour obtenir un indicateur du taux de prévalence du VIH au sein de la filiale, une grande campagne de dépistage a d'abord lieu en 2001 et se répète les années suivantes : la connaissance du statut sérologique des employés est la première étape du programme. Pour des raisons de confidentialité, la campagne de test VCCT (Voluntary Confidential Councelling and Testing) est réalisée par un prestataire de services, Lifeworks. Le directeur général 
participe lui-même au test. De manière similaire, des études « KAP » (Knowledge, Attitude, Practice) sont réalisées régulièrement et portent sur l'ensemble des employés. Ces enquêtes mesurent et évaluent les progrès réalisés ou à réaliser et servent à bâtir les actions conséquentes. Les stratégies sont adaptées selon les résultats de ces enquêtes et selon l'évolution des chiffres de la prévalence du virus.

L'année 2003 correspond à un intense travail de formalisation de la part de la direction générale de Lafarge des politiques définissant la lutte contre le VIH/sida du groupe. Ce travail est réalisé avec les partenaires extérieurs à l'entreprise (l'ONG Care, les syndicats, les fédérations, les organisations internationales, les réseaux professionnels), et trois documents principaux sont publiés en octobre 2003: les directives « santé publique » du groupe; les directives $\mathrm{VIH} / \mathrm{sida}$ du groupe; les directives VIH/sida de la région Afrique. L'entreprise insiste dans ces documents sur les principes de non-discrimination et de confidentialité. Lafarge affirme que «la santé publique est principalement la responsabilité du gouvernement » mais que Lafarge agira en cas d'urgence, notamment si « la contribution de l'entreprise est une partie intégrante des initiatives de la communauté locale ». En Afrique du Sud, les efforts s'orientent vers la communication. Celle-ci se doit d'être créative afin de remettre en cause et de changer les attitudes, les croyances et les pratiques. En 2004, le directeur de la communication dispose d'un budget de 160000 dollars pour mener des actions innovantes et organiser des événements. Dans le but d'éveiller les consciences, on demande aux employés d'assister à un certain nombre d'activités tels que les " industrial theaters ", des animations fondées sur la mise en scène de la maladie. Des témoignages de personnes séropositives et des ateliers sont également organisés. Des préservatifs sont distribués et Lafarge promeut leur utilisation grâce à différentes actions pédagogiques.

Progressivement, au niveau du siège, Lafarge crée des systèmes de gestion pour mettre en application la politique VIH/sida. La direction de la région Afrique conçoit, au début 2004, une feuille de route qu'elle élabore à partir des meilleures pratiques locales. Elle décrit les principales étapes à suivre pour mettre en place un programme $\mathrm{VIH} /$ sida. Les filiales font face à des enjeux typiquement locaux mais on leur demande de mettre en application tous les aspects de la politique VIH/sida globale du groupe. Les expériences des autres entreprises participant au PIA ou à la CME sont très utiles dans l'élaboration de la feuille de route. Pendant les réunions du comité santé Afrique, un tableau de bord est utilisé pour comparer et encourager les filiales sur la base de plusieurs critères. Des visites multiexperts sont organisées et certains directeurs de filiales voient leurs bonus indexés sur la réalisation des objectifs du programme VIH/sida. Un site Intranet est créé et mis à la disposition du comité santé Afrique. Régulièrement mis à jour, il répertorie les comptes rendus des réunions, regroupe les ressources sur la maladie, indique les meilleures expériences et les rend accessibles à chaque filiale africaine. En 2005, la filiale sud-africaine compte 70 «pairs éducateurs ». Ces employés représentent un maillon clé du programme de lutte contre le VIH/sida. Des volontaires de tous les niveaux de l'entreprise et de profils différents (directeurs/employés, noirs/blancs, 
parlant des langues différentes, syndiqués ou pas, hommes/femmes, etc.) reçoivent une formation et du soutien de la part de l'entreprise pour démultiplier l'effort d'éducation, de prévention, d'information, de sensibilisation au sein de l'entreprise. Les pairs éducateurs répondent aux questions des employés. La filiale de Lafarge fournit aussi d'autres structures telles qu'un numéro de téléphone accessible gratuitement par tous les employés. Ce service, géré par un prestataire extérieur, permet aux employés de trouver de l'assistance et du soutien sur les sujets du VIH/sida. Les pairs éducateurs organisent des ateliers au cours de l'année et ils reçoivent, en fin d'année, un certificat personnalisé de la part du directeur général attestant de leur travail bénévole et les remerciant. Depuis juillet 2004, Lafarge Afrique du Sud prend en charge la totalité des frais de traitements pour les employés et leurs épouses, ou leurs partenaires. Si Lafarge assume les coûts des thérapies anti-rétrovirales, le programme inclut aussi la prise en charge d'interventions importantes telles que les conseils diététiques, la fourniture de compléments alimentaires et de vitamines pour les employés concernés par la maladie. Le coût total du programme $\mathrm{VIH} /$ sida représente environ la somme de 500000 dollars par an pour la filiale. Mais, comme l'indique l'audit des coûts réalisé, le coût de l'inaction dépasserait nettement le coût du traitement. En 2006, le département des politiques sociales et le département d'audit du groupe Lafarge travaillent ensemble et mettent au point un référentiel afin de rendre le programme VIH/sida « auditable ». Le groupe cherche également à étendre le programme $\mathrm{VIH} /$ sida à d'autres pays (Russie, Inde et Chine) et à tirer parti de l'expé- rience développée dans la lutte contre le sida en l'appliquant à la gestion d'autres maladies chroniques, tel le paludisme.

En conclusion, cette narration indique que le programme de lutte contre le VIH/sida s'est bâti de manière progressive. Dans un effort de clarification et de synthèse, il est possible de représenter, à l'aide d'un tableau récapitulatif, les principales phases qui caractérisent l'évolution du programme de Lafarge. Les lignes du tableau 1 distinguent ainsi trois niveaux impliqués dans cette politique: la direction générale, la direction de la zone Afrique et la filiale sudafricaine. La monographie fait apparaître la zone Afrique comme un niveau intermédiaire aux relations siège-filiales et qui joue un rôle important dans la construction de la stratégie. Les colonnes correspondent au découpage chronologique du processus en quatre phases. Dans chacune des cellules du tableau, les éléments présentés résument: l'événement (cela correspond à la description de la décision ou de l'action réalisée; figurent par exemple le type de mécanismes de coordination et de contrôle utilisés); les acteurs (les participants clés ainsi que leurs rôles, sont identifiés); les motivations (les raisons de l'action sont précisées); le type de pressions exercées (le contexte de l'action est présenté); le type de flux (les interactions en présence sont distinguées).

Ce tableau est construit à partir de la description de la construction de la stratégie de lutte contre le sida de Lafarge. Il présente un aperçu du processus suivi; il apparaît clairement que différentes phases s'enchaînent: de l'identification d'un enjeu moral au déploiement d'une réponse. Ce tableau est donc particulièrement instructif puisqu'il met en lumière la trajectoire suivie par l'entreprise pour construire sa stratégie de RSE. 
Tableau 1 - Tableau récapitulatif de la construction du programme de lutte contre le VIH/sida de Lafarge

\begin{tabular}{|c|c|c|c|c|}
\hline & Étape $1:$ 2000-2001 & Étape $2:$ 2001-2002 & Étape $3: 2002-2003$ & Étape $4:$ 2003-2005 \\
\hline $\begin{array}{l}\text { Direction } \\
\text { Générale }\end{array}$ & $\begin{array}{l}\text { Événements: Prise de conscience } \\
\text { par le président du problème humain } \\
\text { du sida en Afrique. Il insiste sur les } \\
\text { principes de non discrimination et } \\
\text { du respect de la confidentialité. } \\
\text { Acteurs: Bertrand Collomb. } \\
\text { Motivations: Récente implantation } \\
\text { du groupe en Afrique. } \\
\text { Types de pressions: Faibles sur le } \\
\text { sujet du sida. } \\
\text { Types de flux: Les échanges sont } \\
\text { informels entre la direction générale } \\
\text { et les collaborateurs les plus } \\
\text { impliqués par la problématique du } \\
\text { sida. }\end{array}$ & $\begin{array}{l}\text { Événements: Diagnostic des actions } \\
\text { des filiales. Prises de position du } \\
\text { groupe. Partenariat avec Care. } \\
\text { Audits des coûts en filiales. } \\
\text { Acteurs: Le président et la direction } \\
\text { des politiques sociales. } \\
\text { Motivations: Donner une réponse } \\
\text { homogène et cohérente au problème } \\
\text { du sida au niveau du groupe. Faire } \\
\text { en sorte que toutes les filiales } \\
\text { africaines prennent conscience de } \\
\text { l'enjeu. } \\
\text { Types de pressions: Faibles sur le } \\
\text { sujet du sida mais le groupe les } \\
\text { anticipe. } \\
\text { Types de flux: Ouverture du groupe } \\
\text { vers l'extérieur et recherche } \\
\text { d'informations. Premiers retours } \\
\text { d'expérience du terrain. } \\
\text { Déplacements des dirigeants du } \\
\text { groupe dans les filiales engagées } \\
\text { dans la démarche. }\end{array}$ & $\begin{array}{l}\text { Événements: Nomination d'un } \\
\text { « expert sida » au niveau du groupe. } \\
\text { Création du site Intranet sur le sujet. } \\
\text { Publication des politiques VIH/sida } \\
\text { en octobre 2003. Recherche de } \\
\text { partenaires. } \\
\text { Acteurs: La direction des politiques } \\
\text { sociales. } \\
\text { Motivations: Réussir à convaincre } \\
\text { les filiales de s'engager dans la lutte } \\
\text { contre le sida. } \\
\text { Types de pressions: De plus en plus } \\
\text { fortes de la part des parties } \\
\text { prenantes du groupe. } \\
\text { Types de flux: Interactions } \\
\text { fréquentes entre la direction } \\
\text { générale, la zone Afrique et les } \\
\text { filiales sur le sujet du sida. }\end{array}$ & $\begin{array}{l}\text { Événements : Élaboration du tableau } \\
\text { de bord et de la feuille de route. } \\
\text { Rendre le programme « auditable ». } \\
\text { Introduction du sida comme un } \\
\text { critère dans les programmes de } \\
\text { performance du groupe. } \\
\text { Organisation de visites multi } \\
\text { experts. Communications et } \\
\text { publications sur le sujet. } \\
\text { Acteurs: La direction des politiques } \\
\text { sociales. } \\
\text { Motivations: Pérenniser le } \\
\text { programme. } \\
\text { Types de pressions: De plus en plus } \\
\text { fortes de la part des parties } \\
\text { prenantes du groupe. } \\
\text { Types de flux: Interactions régulières } \\
\text { avec la zone et les filiales. }\end{array}$ \\
\hline Zone & & $\begin{array}{l}\text { Evénements: Lancement d'un projet } \\
\text { pilote pour toutes les filiales } \\
\text { africaines. } \\
\text { Acteurs: Le président de la zone } \\
\text { Afrique, Tony Hadley, porte le } \\
\text { projet. } \\
\text { Motivations: Économiques et } \\
\text { sociales: le sida fait peser un risque } \\
\text { sur la pérennité des activités des } \\
\text { filiales africaines. }\end{array}$ & $\begin{array}{l}\text { Événements: Création du comité } \\
\text { santé Afrique, organisation des } \\
\text { réunions et suivi. } \\
\text { Acteurs: Le président de la zone } \\
\text { Afrique et le DRH de la zone. } \\
\text { Motivations: Valoriser les filiales les } \\
\text { plus en avance, inciter les autres. }\end{array}$ & $\begin{array}{l}\text { Événements: Choix des priorités et } \\
\text { du rythme de mise en œuvre. Bonus } \\
\text { accordés aux dirigeants de filiales } \\
\text { sur le sujet du sida. } \\
\text { Acteurs: Le président Afrique. } \\
\text { Motivations: Arbitrage entre les } \\
\text { priorités. }\end{array}$ \\
\hline
\end{tabular}




\begin{tabular}{|c|c|c|c|c|}
\hline & Étape $1: 2000-2001$ & Étape 2: 2001-2002 & Étape 3: 2002-2003 & Étape 4: 2003-2005 \\
\hline Zone & & $\begin{array}{l}\text { Types de pressions: Celles des } \\
\text { filiales et celles de la direction } \\
\text { générale. } \\
\text { Types de flux: Rencontres fréquentes } \\
\text { avec les filiales et avec la direction } \\
\text { générale. }\end{array}$ & $\begin{array}{l}\text { Types de pressions: Économiques et } \\
\text { sociales. } \\
\text { Types de flux: Rencontres formelles, } \\
\text { organisées par les réunions du } \\
\text { comité santé. }\end{array}$ & $\begin{array}{l}\text { Types de pressions: Concilier les } \\
\text { pressions de rentabilité à court } \\
\text { terme des filiales avec les pressions } \\
\text { du groupe. } \\
\text { Types de flux: Discussions et } \\
\text { négociations avec les filiales. }\end{array}$ \\
\hline Filiales & $\begin{array}{l}\text { Événements: Actions disparates de } \\
\text { lutte contre le sida au sein de } \\
\text { certaines filiales africaines. } \\
\text { Acteurs: Le dirigeant de la filiale. } \\
\text { Motivations: Perte de main d'œuvre, } \\
\text { le moral des équipes se dégrade, } \\
\text { enjeu moral. } \\
\text { Types de pressions: Faibles } \\
\text { pressions RSE de la part du contexte } \\
\text { local. } \\
\text { Fortes pressions au niveau } \\
\text { économique. } \\
\text { Types de flux: Très peu de contacts } \\
\text { avec la direction générale sur le } \\
\text { sujet du sida. }\end{array}$ & $\begin{array}{l}\text { Événements: Expérimentations: la } \\
\text { filiale cherche à définir son plan } \\
\text { d'actions. Campagne d'information } \\
\text { et de sensibilisation. Testing et étude } \\
\text { sur les comportements des employés } \\
\text { en Afrique du Sud. } \\
\text { Acteurs: Fort leadership du dirigeant } \\
\text { de la filiale qui soutient le projet et } \\
\text { qui participe aux opérations. } \\
\text { Motivations: Volonté d'apporter une } \\
\text { réponse concrète au problème du } \\
\text { sida. } \\
\text { Types de pressions: Forte prise en } \\
\text { compte des caractéristiques locales: } \\
\text { croyances locales, obstacles } \\
\text { culturels et historiques, etc. } \\
\text { Types de flux: Les filiales } \\
\text { développent leurs projets de manière } \\
\text { autonome. Forte interaction avec } \\
\text { l'environnement local. }\end{array}$ & $\begin{array}{l}\text { Événements: Actions de plus en plus } \\
\text { structurées. Innovation et } \\
\text { créativité dans les actions } \\
\text { (« industrial theaters », etc.). } \\
\text { Importante opération de } \\
\text { communication et de sensibilisation } \\
\text { en Afrique du Sud. Mise en place du } \\
\text { système de «pairs éducateurs ». } \\
\text { Acteurs: Fort leadership du dirigeant } \\
\text { de la filiale. Constitution d'une } \\
\text { équipe locale dans la filiale en } \\
\text { charge du programme sida. } \\
\text { Motivations: Définir la bonne } \\
\text { méthode de combattre le sida pour } \\
\text { obtenir des résultats localement. } \\
\text { Types de pressions: Forte prise en } \\
\text { compte des caractéristiques locales. } \\
\text { Types de flux: Interactions avec la } \\
\text { direction générale et les autres } \\
\text { filiales (partage d'expérience). }\end{array}$ & $\begin{array}{l}\text { Événements: En juillet 2004, prise } \\
\text { en charge des traitements des } \\
\text { employés séropositifs par la filiale } \\
\text { sud-africaine. } \\
\text { Acteurs: Le comité exécutif de la } \\
\text { filiale est impliqué dans ce type de } \\
\text { décisions. } \\
\text { Motivations: Étendre l'expérience } \\
\text { acquise à d'autres problématiques } \\
\text { (le paludisme par exemple). } \\
\text { Types de pressions: D'autres } \\
\text { problématiques locales émergent. } \\
\text { Types de flux: La marge de } \\
\text { manœuvre des filiales est moins } \\
\text { forte sur le sujet du sida qu'au début } \\
\text { du processus. }\end{array}$ \\
\hline
\end{tabular}


Il convient maintenant d'analyser et de caractériser plus en détail le type de processus dont il s'agit.

\section{III - UN PROCESSUS \\ TRANSNATIONAL DE \\ CONSTRUCTION DE STRATÉGIE \\ DE RESPONSABILITÉ SOCIALE DANS LA FIRME MULTINATIONALE}

Cette troisième partie introduit les fondements de l'analyse de processus stratégiques et propose une conceptualisation $\mathrm{du}$ processus observé dans le but d'en extraire un sens générique qui dépasse le simple contexte du cas Lafarge et de la lutte contre le sida.

\section{Une chronologie de la stratégie: déclenchement - conception - structuration - généralisation}

La littérature stratégique admet généralement un découpage des processus stratégiques en trois ou quatre phases principales. Que ce soit les travaux qui portent sur la réflexion stratégique et sur les processus stratégiques (Bower, 1970; Torset, 2002), sur les processus de décision (Mintzberg et al., 1976), sur les processus d'innovation (Burgelman, 1983, 1991) ou sur les processus de redirection stratégique et de changement stratégique (Doz et Prahalad, 1987), tous expriment une même séquence: un projet est d'abord initié, puis développé, avant d'être rationalisé et enfin mis en œuvre dans l'entreprise. En ce qui concerne l'innovation, Burgelman propose un processus de l'intraprise construit autour de quatre phases qui s'enchaînent: il distingue ainsi les phases de « définition », de " poussée ", de " contexte stratégique " et de « contexte structurel ». La question est de savoir si les stratégies de RSE suivent une même logique de construction dans la firme multinationale. À la lecture de la monographie du cas Lafarge, et notamment du tableau récapitulatif, le lecteur peut suivre la trajectoire de construction du programme de lutte contre le sida, laquelle fait apparaître, de manière dynamique, quatre phases principales qui se succèdent. Il est ainsi possible de distinguer d'une part, la formulation de la stratégie et d'autre part, sa mise en œuvre. La formulation correspond aux deux premières phases du tableau 1, que l'on peut nommer le « déclenchement » et la « conception » de la stratégie, tandis que la mise en œuvre correspond aux deux dernières phases, la « structuration » et la « généralisation » de la stratégie. On retrouve alors la même logique du découpage en quatre phases présentée dans les travaux de Burgelman (1983, 1991). À ce stade de la recherche, il est possible d'aller plus en profondeur dans l'effort de conceptualisation du processus de construction de la stratégie de RSE et de décrire les significations conférées à chacune des quatre phases identifiées. Il devient alors envisageable de s'échapper du contexte spécifique de la lutte contre le sida et de proposer un processus potentiellement générique de construction de stratégie RSE.

\section{Un modèle séquentiel et interactif}

Le tableau 2 nomme les actions de chaque phase et offre une présentation simplifiée du processus. Le contenu de chacune des cellules est ensuite discuté.

Le déclenchement $\gg$ du processus se caractérise simultanément par une impulsion de la direction générale et par la découverte, par les filiales, d'un enjeu local nouveau. La direction de la zone ne joue pas encore, durant cette phase initiale, de rôle majeur. 
Tableau 2 - Processus transnational de construction de stratégie de responsabilité sociale dans la firme multinationale

\begin{tabular}{|c|c|c|c|c|}
\hline & \multicolumn{2}{|c|}{ Formation } & \multicolumn{2}{|c|}{ Mise en ouvre } \\
\hline & Déclenchement & $\longrightarrow$ Conception & Structuration & $\longrightarrow$ Généralisation \\
\hline $\begin{array}{l}\text { Direction } \\
\text { générale }\end{array}$ & $\begin{array}{c}\text { IMPULSION } \\
\text { Identification d'un } \\
\text { enjeu moral global } \\
\text { Le leader }\end{array}$ & $\begin{array}{c}\text { RÉFLEXION } \\
\text { Collection des } \\
\text { initiatives des filiales }\end{array}$ & $\begin{array}{l}\text { FORMALISATION } \\
\text { Construction d'une } \\
\text { valeur ajoutée } \\
\text { Le sponsor }\end{array}$ & $\begin{array}{l}\text { ANCRAGE } \\
\text { Intégration } \\
\text { de la stratégie }\end{array}$ \\
\hline Zone & - & $\begin{array}{l}\text { CRISTALLISATION } \\
\text { Transformation en } \\
\text { enjeu économique } \\
\text { Le champion }\end{array}$ & $\begin{array}{l}\text { ÉMULATION } \\
\text { Organisation d'un } \\
\text { climat de } \\
\text { stimulation }\end{array}$ & $\begin{array}{l}\text { MISE EN CEUVRE } \\
\text { Organisation } \\
\text { du rythme } \\
\text { et des priorités }\end{array}$ \\
\hline Filiale & $\begin{array}{l}\text { DÉCOUVERTE } \\
\text { Identification d'un } \\
\text { enjeu moral local } \\
\text { L'entrepreneur } \\
\text { responsable }\end{array}$ & $\begin{array}{c}\text { EXPÉRIMENTATION } \\
\text { Amplification } \\
\text { d'actions locales } \\
\text { hétérogènes }\end{array}$ & $\begin{array}{l}\text { ACTION } \\
\text { Innovation } \\
\text { et expression } \\
\text { de créativité }\end{array}$ & $\begin{array}{l}\text { HOMOGÉNÉISATION } \\
\text { Pérennisation } \\
\text { des actions }\end{array}$ \\
\hline
\end{tabular}

Le rôle de « leadership » exercé au sommet de la hiérarchie apparaît comme étant essentiel au démarrage du processus. Le dirigeant local est qualifié « d'entrepreneur responsable » car c'est lui seul, à ce stade du développement de la stratégie, qui choisit d'impliquer ou non son entreprise.

La « conception » de la stratégie se réalise par le biais d'un travail de réflexion de la part de la direction générale qui s'accompagne d'expérimentations menées dans les filiales. La direction de zone joue alors un rôle capital de cristallisation et de transformation de l'enjeu social et humain en enjeu économique. Le dirigeant de la zone, qui est nommé " champion » de la stratégie, sert ainsi de relais et d'intermédiaire entre les préoccupations concrètes des filiales et l'ambition de la direction générale. Cette rencontre s'organise grâce au dirigeant de la zone, ce qui est particulièrement important dans la mesure où c'est souvent cet acteur qui possède le pouvoir exécutif. La firme multinationale est en effet un lieu où s'exercent des antagonismes, où s'expriment des tensions et se ressentent des conflits (Dörrenbächer et Geppert, 2006). C'est un espace politique où les individus, ou les groupes d'individus, peuvent revendiquer des intérêts potentiellement divergents: les filiales reçoivent parfois des injonctions contradictoires ou paradoxales de la part de la direction générale et/ou de la part de la direction de zone; cela rend la formulation et la mise en œuvre de stratégies d'autant plus difficile (Birkinshaw et Ridderstrale, 1999). Le «champion » apparaît ici comme celui qui réduit ces divergences et facilite la déclinaison de la stratégie RSE au niveau local; il rapproche 
notamment la préoccupation de comportement socialement responsable avec les objectifs et exigences de profitabilité.

Ensuite, la «structuration » de la stratégie s'effectue grâce aux efforts de formalisation de la stratégie opérés à la direction générale alors que la zone organise un climat d'émulation entre les filiales qui ellesmêmes entreprennent des actions locales concrètes. La direction générale renforce son expertise vis-à-vis des filiales en étoffant ses ressources et en recrutant une personne ressource que l'on peut qualifier de « sponsor» de la stratégie.

Finalement, la « généralisation » de la stratégie correspond à un ancrage dans le contexte structurel plus traditionnel de l'entreprise et la mise en cuvre de la stratégie est organisée directement par la zone alors que les actions des filiales suivent une tendance d'homogénéisation.

\section{CONCLUSION}

Le processus de construction de stratégie RSE proposé dans cette recherche fait ressortir un fort degré d'interaction entre les trois principaux éléments constitutifs d'une firme multinationale: le siège, la zone et la filiale. La stratégie ne résulte ni de l'initiative d'une filiale, ni d'une volonté délibérée de la direction générale. Autrement dit, il ne s'agit ni d'un processus uniquement ascendant (bottom-up), ni strictement descendant (top-down). Le phénomène observé s'apparente plutôt à une construction hybride, transnationale, mixte, interactive, qui fait intervenir simultanément les différents niveaux de la firme. Cette recherche confirme ainsi l'existence de stratégies de RSE hybrides, ou transnationales comme le nomme Arthaud-Day (2005). L'article met en avant l'intérêt et la nécessité de dépasser le clivage entre RSE locale et RSE globale en positionnant la réflexion entre le relativisme éthique et l'impérialisme éthique. La résolution de dilemme d'ordre éthique est facilitée par une succession de phases de tâtonnement, d'expérimentation locale, d'incitation et de structuration; quatre acteurs apparaissent comme rouages essentiels à la construction de la stratégie.

D'un point de vue managérial, cette recherche propose aux dirigeants de les guider dans les différentes phases de construction de leurs stratégies de RSE. Il ne s'agit pas de fournir des solutions ou des référentiels de bonnes pratiques à copier, cela ne fonctionnerait pas compte tenu, d'une part, de la diversité des problématiques de RSE et, d'autre part, de la complexité de la gestion du local et du global dans la firme multinationale. Bien que l'étude de cas unique limite la portée de généralisation des résultats, l'approche processuelle a le mérite de proposer une logique de raisonnement qui permet à chaque firme de concevoir ses propres stratégies de RSE en tenant compte des particularités de son contexte organisationnel.

Le processus transnational proposé fournit une première brique dans la conceptualisation du phénomène de construction de stratégies de RSE; il interroge également le caractère spécifique des firmes multinationales françaises. Les travaux de recherche récents portant sur le management international analysent en effet l'impact de ces spécificités dans les choix de localisation des activités (Colovic et Mayrhofer, 2008), dans l'explication des performances managériales et organisationnelles (Josserand, 2004; Collin et Rouach, 2009), ou dans l'élaboration de stratégies internationales 
(Pestre, 2011). Cet article ouvre la voie à des investigations comparatives destinées à mieux comprendre l'impact de la culture d'origine des firmes sur la détermination et la construction de leurs stratégies RSE. Il est ainsi probable que les firmes anglosaxonnes se distinguent dans le degré de formalisation de ces stratégies, qu'elles utilisent d'autres mécanismes de coordination et de contrôle et qu'elles accordent une marge d'autonomie différente à leurs filiales. Ce travail s'inscrit donc dans un programme de recherche plus vaste et invite à poursuivre la théorisation en répondant à la question suivante: existe-il, dans les firmes multinationales, des processus de construction de stratégies de RSE descendants, c'est-à-dire impulsés par la direction générale, et ascendants, c'est-à-dire initiés par la filiale?

\section{BibliograPHIE}

Aggeri F., Pezet E., Acquier A., Organiser le développement durable: Expériences des entreprises pionnières et formation de règles d'action collective, Vuibert, Paris, 2005.

Arthaud-Day M.L., "Transnational corporate social responsibility: A tri-dimensional approach to international CSR research", Business Ethics Quarterly, vol. 15, n 1, 2005, p. $1-22$.

Beddi H., Contribution à l'analyse de la diversité des relations sièges-filiales au sein des firmes multinationales, Thèse de doctorat, Université Paris-Dauphine, Paris, 2008.

Birkinshaw J.M., Hood N. (Eds.), Multinational corporate evolution and subsidiary development, MacMillan Press, London, 1998.

Birkinshaw J., Ridderstrale J., "Fighting the corporate immune system: A process study of subsidiary initiatives in multinational corporations", International Business Review, vol. 8, $\mathrm{n}^{\circ} 2,1999$, p. 149-180.

Bower J.L., Managing the resource allocation process: A study of corporate planning and investment, Harvard Business School Press, Boston, Mass, 1970.

Burgelman R.A., "A process model of internal corporate venturing in diversified major firm”, Administrative Science Quarterly, vol. 28, n 2, 1983, p. 223-244.

Burgelman R.A., "Intraorganizational ecology of strategy making and organizational adaptation: Theory and field research", Organization Science, vol. 12, n 3, 1991, p. 239-262.

Capron M., Quairel F., La responsabilité sociale des entreprises, coll. « Repères », La Découverte, Paris, 2007.

Collin B., Rouach D., Le modèle L'oréal. Les stratégies clés d'une multinationale française, Pearson Education, 2009.

Colovic A., Mayrhofer U., « Les stratégies de localisation des firmes multinationales: une analyse du secteur automobile », Revue française de gestion, vol. 34, $\mathrm{n}^{\circ} 184,2008$, p. 151-165.

Donaldson T., "Values in tension: Ethics away from home", Harvard Business Review, septembre/octobre 1996, p. 48-62. 
Dörrenbächer C., Geppert M., "Micro-politics and conflicts in multinational corporations: current debates, re-framing, and contributions of this special issue", Journal of International Management, vol. 12, $\mathrm{n}^{\circ}$ 3, 2006, p. 251-265.

Doz Y.L., Prahalad C.K., "A process model of strategic redirection in large complex firms: The case of multinational corporations", The management of strategic change, Pettigrew A. (Eds.), Basil Blackwell, Oxford, England, p. 63-88, 1987.

Egelhoff W.G., "Information-processing theory and the multinational enterprise", Journal of International Business Studies, vol. 22, n 3, 1991, p. 341-368.

Ghemawat P., "Reconceptualizing international strategy and organization", Strategic Organization, vol. 6, $\mathrm{n}^{\circ}$ 2, 2008, p. 195-206.

Goold M., Campbell A., Strategies and styles: The role of the centre in managing diversified corporations, Basil Blackwell, Oxford, 1987.

Gordon K., « Des normes de comportement mondial pour les entreprises? », Revue française de gestion, vol. 136, nov.-déc. 2001, p. 26-34.

Husted B.W., Allen D.B., "Corporate social responsibility in the multinational enterprise: Strategic and institutional approaches", Journal of International Business Studies, vol. 37, $\mathrm{n}^{\circ} 6,2006$, p. 838-849.

Josserand E., The network organization. The experience of leading French multinationals, Edward Elgar, Cheltenham, 2004.

Logsdon J.M., Wood D.J., "Business citizenship: From domestic to global level of analysis", Business Ethics Quarterly, vol. 12, n² 2, 2002, p. 155-187.

Martinez J.I., Jarillo J.C., "The evolution of research on coordination mechanisms in multinational corporations”, Journal of International Business Studies, vol. 20, n 3, 1989, p. 489-514.

Martinet A.-C., Reynaud E., Stratégie d'entreprise et écologie, Economica, Paris, 2004.

Martinet A.-C., Payaud M.A., «Le développement durable, vecteur et produit d'une régénération de la gouvernance et du management stratégique: Un cadre théorique intégrateur », Management International, vol. 12, $\mathrm{n}^{\circ}$ 2, 2008, p. 13-25.

Meyer K.E., "Perspectives on multinational enterprises in emerging countries", Journal of International Business Studies, vol. 35, n 4, 2004, p. 259-276.

Mintzberg H., Raisinghani D., Théorêt A., "The structure of 'unstructured' decision processes", Administrative Science Quarterly, vol. 21, n 2, 1976, p. 246-275.

O’Donnell S.W., "Managing foreign subsidiaries: Agents of headquarters, or an interdependent network?", Strategic Management Journal, vol. 21, n 5, 2000, p. 525-548.

Otterbeck L., The management of headquarters-subsidiary relations in multinational corporations, Gower, Aldershot, 1981.

Persais E., « La gestion de la RSE dans un contexte international: vers une "glocalisation" des pratiques? Une étude de cas d'entreprises françaises implantées au Mexique », Management International, vol. 14, $\mathrm{n}^{\circ}$ 2, 2010, p. 11-30.

Pestre F., «Les spécificités de la mise en œuvre de stratégies de RSE à l'international », RSE : Regards croisés Droit et Gestion, Economica, Paris, à paraître, 2011. 
Pisani N., "International management research: Investigating its recent diffusion in top management journals", Journal of Management, vol. 35, n² 2, 2009, p. 199-218.

Prahalad C.K., Doz Y.L., The multinational mission: Balancing local demands and global vision, The Free Press, New York, 1987.

Torset C., La décentralisation de la réflexion stratégique, Thèse de doctorat, Université ParisDauphine, Paris, 2002.

Yin R.K., Case study research: Design and methods (3rd ed.), Sage, Thousand Oaks, CA, 2003. 
